\title{
Regional Anesthesia Procedure
}

National Cancer Institute

\section{Source}

National Cancer Institute. Regional Anesthesia Procedure. NCI Thesaurus. Code C64381.

The administration of pharmaceutical agents intended to produce loss of sensation in a particular anatomical region. 